\title{
Review on waste heat utilization technology of circulating water in power plant Ning Dong ${ }^{1, a}$
}

\author{
${ }^{1}$ School of Energy Power and Mechanical Engineering,North China Electric Power \\ University,Baoding 071000,China \\ a2991415515@qq.com
}

Keywords: Circulating water in power plant, surplus heat utilization, heat pump, The smoke tower in one, coarse vacuum.

\begin{abstract}
Circulating water in thermal power plant as a unit of the cooling medium, takes a lot of heat and released into the environment, not only causes environmental pollution but also makes that the resource utilization rate of thermal power plant is not high. Through the water source heat pump technology, the smoke tower technology and the steam turbine low vacuum operation technology make full use of thermal power plant circulating water waste heat, which are of great significance of China's energy-saving emission reduction.
\end{abstract}

\section{Introduction}

With the rapid development of China's economy, all walks of life between the growing demand for energy and energy reserves are increasingly less, so the implementation of energy-saving emission reduction for sustainable development is of great significance. In the context of energy saving and emission reduction, the large amount of waste heat in the circulating water of thermal power plant has not been fully utilized and has attracted the attention of people.

At present, in China's large and medium-sized cities in widespread centralized heat supply can not meet the demand for heating, some places in order to alleviate the situation of heating tension, the blind development of small coal-fired boiler room, gas heating and electric heating, not only the heating cost and deteriorates the atmospheric environment. Therefore, if the waste heat of circulating cooling water for heating (hot water heating, etc.), not only can reduce the environmental pollution of thermal power plant, and can alleviate the tense situation of heating.

Power plant circulating water is used for heating in the presence of mainly of circulating water temperature is low, it is difficult to achieve the direct heating of the grade requirements, should be appropriate to raise the temperature of the circulating water, the method currently used in most of there are two: one is reduce the exhaust cylinder vacuum degree and improve the exhaust steam temperature, steam turbine in low vacuum operation; the second is to power plant circulating water as a low heat, the heat pump technology absorbs the waste heat to achieve heating. In addition, the circulating water can also play a role in purifying flue gas.

\section{Low vacuum heat supply technology for steam turbine}

The operating principle of low vacuum operation of the steam turbine, is appropriate to reduce the vacuum of condenser, steam turbine exhaust pressure increases, improve the exhaust steam temperature, so as to improve the temperature of the circulating cooling water, condenser circulating water can be directly as a heating medium in heat supply system in closed loop. Low vacuum operation of steam turbine, the condenser is equivalent to the original heating network. Figure 1 is a simple system of steam turbine low vacuum circulating water heating.

Traditional low vacuum heat supply mode. There are two limitations in the traditional low vacuum heat supply technology, namely, the separation of power generation and heat load, and the transformation of the steam turbine unit. First, through the traditional low vacuum operation of the unit steam consumption is dependent on the size of the heat load of users, power generation unit by 
constraints on the user 's heat load size, not free and independent regulation, it is only applicable to the heating system to heat the user load is relatively stable. Secondly, the transformation of steam turbine units into low vacuum operation and required after varying operating conditions to calculate the exhaust cylinder structure, the axial thrust change, the transformation of the last stage impeller, make a strict examination and certain changes when it can be implemented, this is true only in a small and a medium sized unit, for the reheat steam turbine generator sets, high condenser pressure will enable the steam outlet of the last stage of high temperature and steam flow is too small, resulting in strong vibration of the unit, endanger the safe operation of the unit.

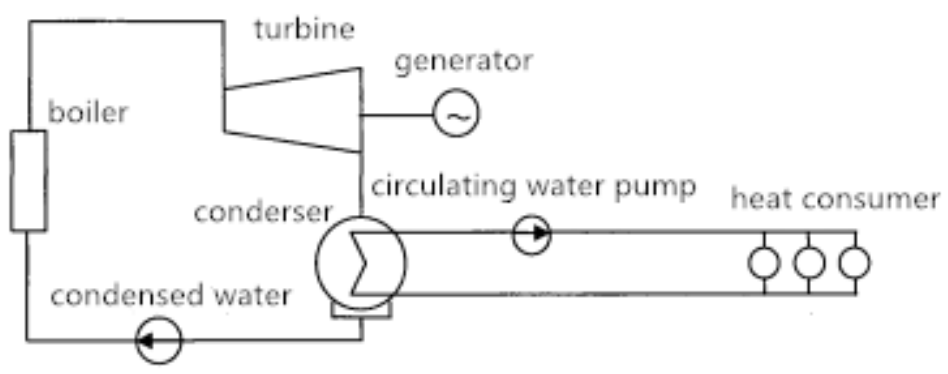

Fig. 1 Simple system of low vacuum circulating water heating for steam turbine

Low vacuum operation low temperature heat supply mode. There is one kind of low vacuum operation mode which is suitable for large scale units, that is, the steam exhaust pressure of large units is not more than the design value, and the circulating water of about 40 degrees Celsius is supplied directly to the heating system. When the heat load of condenser is larger than the demand heat load, the excess heat load is discharged into the environment, which can realize the independent regulation of the thermal load and the thermal efficiency is the highest. This kind of heating mode is called low vacuum operation low temperature heat supply mode.

Although the normal and safe operation of the low vacuum operation of low temperature heating mode does not affect the steam turbine and power generation of unit not by the heat load of the user control, and is also suitable for small, medium and large units, but this heating method exists some disadvantages: heating temperature is too low, not suitable for the end of high demand for heating; the second is the temperature difference is small, small temperature difference, high flow will increase transportation energy consumption in the process, which restricts the heating radius. So it can make the whole comprehensive energy consumption and economy be affected.

"NCB" new large-capacity Heating Unit."NCB" new special heating backpressure steam turbine has both advantages and extraction-condensing heating units, and at the same time overcome the shortcomings of the two heating units. Characterized by the extraction condensing heating machine based on the use of two shafts are driven by two generators, shown in Figure 2.

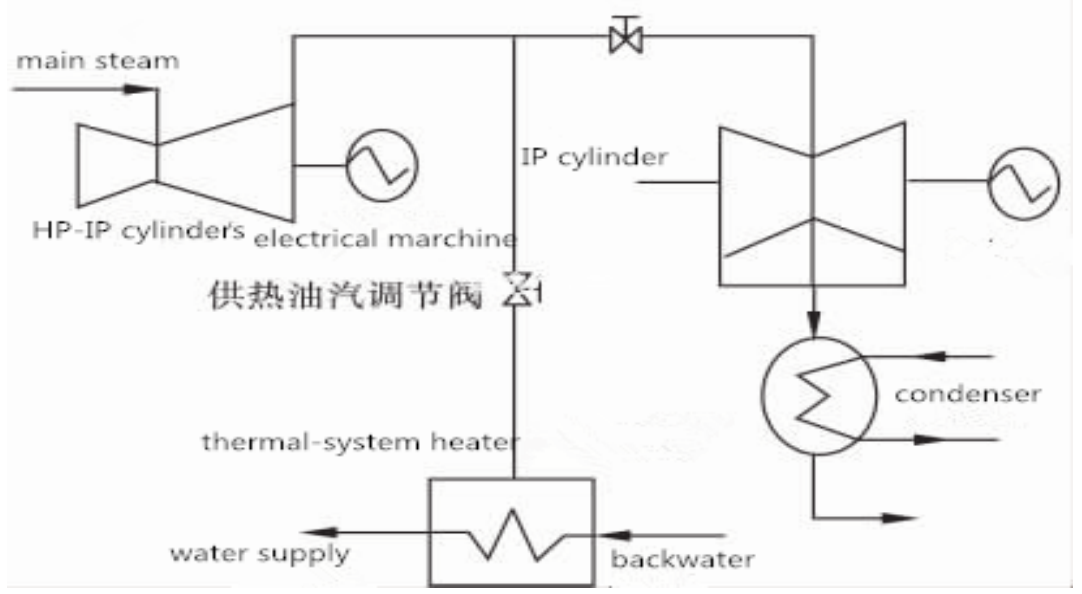

Fig. 2 "NCB" new heating unit working process

Non heating, heating oil steam control valve is fully closed, the low-pressure cylinder control valve fully open, steam turbine condition of pure condensate, has the advantages of pure condensing steam turbine high efficiency power generation; normal heating, heating oil steam regulating valve 
and the low pressure cylinder control valve in the regulation of the state, steam turbine for extraction steam conditions, with extraction condensing steam turbine of external pumping steam heating and keep the advantages of high power generation efficiency; peak heating period, heating oil steam regulating valve fully open, the low-pressure cylinder adjusting valve is fully closed, steam turbine back pressure conditions, with advantages of back pressure steam turbine. The whole unit has a high thermal efficiency in the non - heating period and the heating period.

"NCB" new heating units to maximize the use of a moderate grade high pressure cylinder exhaust, greatly reducing the loss of the cold source. This technology has many advantages, but it is still in the stage of theoretical research, and it is still needed to consider more technical risk problems in the field.

\section{Heat pump recovery waste heat technology}

The waste heat of circulating water in thermal power plant is a kind of low grade energy, the efficiency and scope of the direct use is very low. However, if the use of heat pump technology, can effectively improve the circulating cooling water quality, expand the scope of its use, and thus improve the efficiency of the use of. Heat pump technology in recent years in our country has been widely used, can be absorbed, the use of steam, gas and so on as the driving source of heat, can also be compressed, the use of power as a driving source.

Compression type heat pump heating mode.Compression type heat pump heating technology into distributed electric heat pump heating and centralized electric heat pump heating mode.

The technology of distributed electric heating heat pump, heat pump located in the district heating station, thermal power station in the heat pump circulating water heat heating net two times water to provide hot water and heating to the user. This way has a certain energy-saving effect, but pipeline investment is large, conveying high consumption, heating range only around the plant within 3 to 5 $\mathrm{km}$. The flow chart of the distributed electric heat pump heating system is shown in Figure 4.

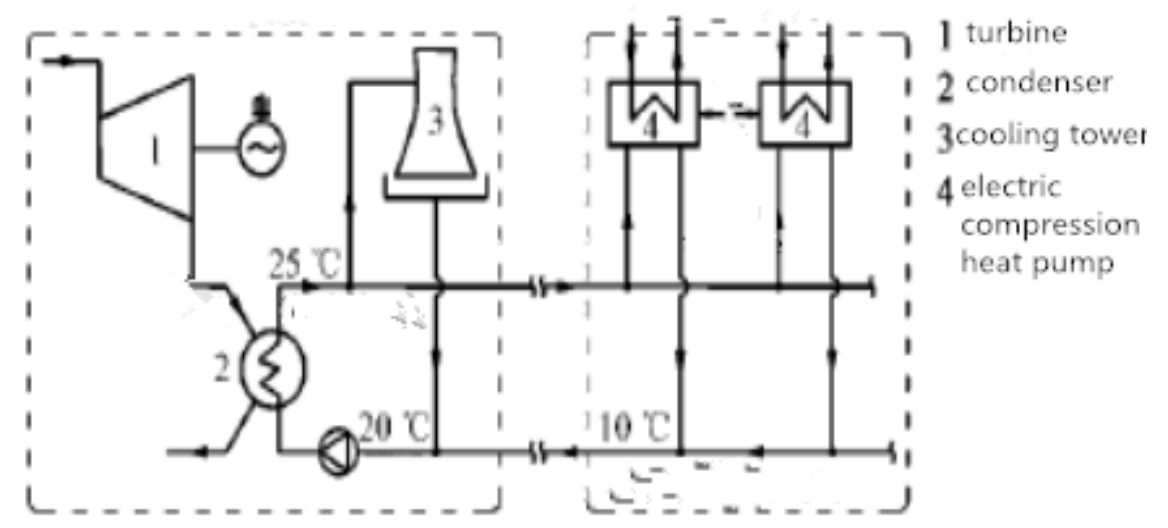

Fig. 3 Two or more references

Centralized electric heat pump technology, heat pump unit is located in the power plant, condenser outlet portion of the circulating water as low temperature heat source into heat pump, heating a water network to 80 to 90 DEG C, the heat exchanger for secondary heating, into the urban heating network. This way of heating the initial investment is small, but the electric power plant, power plant electricity capacity to bring pressure. Centralized heating system flow Figure 4 as shown below.

Central absorption heat pump heating mode.Absorption heat pump unit inside the plant, process and centralized electric heat pump heating in a manner similar to, by use of steam turbine extraction steam as a heat pump driven heat source, spent steam waste heat as low temperature heat source heat pump, heating a water network to 80 DEG C about output, and the peak heater for heating.

This method can use some or all of the steam turbine exhaust waste heat, energy saving effect is better. At present, the use of centralized absorption heat pump technology is the most widely used in thermal power plant waste heat recovery technology. 


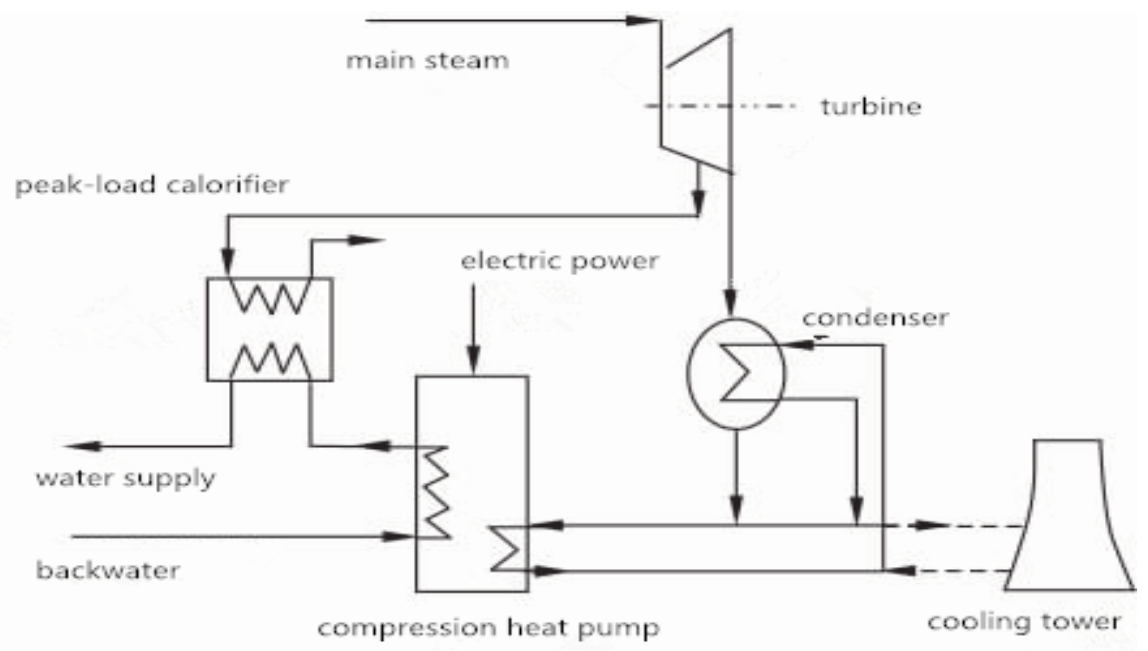

Fig. 4 Central electric heat pump heating system flow

\section{Combined technology of flue gas tower in power station}

In the thermal power plant flue gas temperature after desulfurization in the vicinity of the saturation temperature, if the chimney emissions, there are some difficulties. Because after desulfurization of flue gas temperature is low, after the heat dissipation of the chimney the flue gas temperature is lower, the uplift of the flue gas is highly affected by the larger limit, this will lead to the power plant near the ground with high concentration of pollutants, is not conducive to environmental protection requirements.

After the net flue gas desulfurization can be directly through the cooling tower emissions, the huge cooling tower thermal wet air wrapped around the net flue gas, increase the lifting height of the flue gas, so as to promote the flue gas diffusion, namely the smoke tower synthetic new technology.

Smoke tower integrated technology can save the initial investment is a new power plant, save the chimney, flue gas reheater and desulfurization booster fan to the cost of investment, but also increased the increase in cooling tower anticorrosion, flue and water consumption of new investment, the key is can increase the lifting height of the flue gas emissions, conducive to the spread of smoke and reduce pollution near power plants.

\section{Summary and recommendations}

(1) the traditional low vacuum operation heating technology of steam turbine does not apply to modern high-capacity, high parameter units.However, low vacuum operation of low temperature heating, "the NCB heating units, such as technology, expand the application width of low vacuum operation of the steam turbine heating technology.

(2) heat pump heat recovery technology, could flexibly adjust the relationship between the heating load and user, which is a ideal way recycling power plant circulating water waste heat for heating. But, practical application need to consider the energy efficiency, economic cost, heat transmission and other problems, select the appropriate form of system.

(3) smoke tower technology, not only can improve the energy efficiency of thermal power plant, but also greatly simplify the thermal power plant flue gas system, reduce equipment investment, can get better economic benefits and environmental benefits.

(4) proposed by Tsinghua University in 2007 "based on the absorption cycle cogeneration central heating", to improve the ability of heating, increasing the network transmission capacity and power plant energy efficiency effectively combine, is a technical innovation in cogeneration central heating area.

(5) the use of circulating water of power plant waste heat is mostly used in the northern city of heating and hot water supply. In view of the characteristics of summer high temperature in part of the southern region and long duration,it can be part of the focus of the work back in using heat pump 
technology to recycle the circulating water of power plant waste heat, driven absorption chiller cooling capacity for, but also need from two aspects of the technical and economic feasibility analysis in China"s southern region recycling thermal power plant circulating water heat for focus for the feasibility of the cold.

\section{References}

[1]Weiwei Bao, Wei Ren, Qilin Zhang. Analysis of the characteristics of low vacuum heat supply for large air cooling units [J]. district heating, 2015,04:73-78+124.

[2] Jie Zheng. Application of [J]. energy saving technology in steam turbine low vacuum circulating water heating technology, 2006,04:380-382.

[3] Jianren He, Damao Xu. Thermoelectric technology NCB new special heating machine [J]. energy efficiency, 2009,03:1-4+11.

[4] Baoyu Wang, Chongbo Zhou. A heat pump system of thermal power plant circulating water heat of [J]. modern electric power, 2011,04:73-77.

[5] Xuelei Zhang, Haiping Chen. Thermal performance analysis of heat pump system with waste heat recovery of circulating water [J]. proceedings of the China Electrical Engineering, 2013,08:1-8+15.

[6] Yueming Liang. Research and analysis of the technology of smoke column in [D]. North China Electric Power University (Beijing), 2007.

[7] Lin Fu, Yi Jiang, Shigang Zhang. A method of combined heat and power generation based on Co-ah cycle $[\mathrm{J}]$. Journal of Tsinghua University (NATURAL SCIENCE EDITION), 2008,09:1377-1380+1412.

[8] Yan Yi, Lin Fu, Shigang Zhang, Hongfa Di, Changlei Xiao. Power plant circulating water waste heat utilization technology review [a]. The Architectural Society of China HVAC branch, Chinese Association of refrigeration air conditioning heat pump professional committee. National HVAC refrigeration 2010 academic year will Symposium [C]. China Construction Building Society HVAC branch, Chinese Association of refrigeration air conditioning heat pump professional committee: 2010:5.

[9] Feng Wang. Study on the waste heat utilization scheme of circulating water in power plant [D]. North China Electric Power University (Beijing), 2010. 